\title{
Numerical study into the breakdown strength of a two phase (gas-liquid) insulation system
}

\author{
S Wu*, R. D. Chippendale, P. L. Lewin \\ The Tony Davies High Voltage Laboratory \\ University Of Southampton \\ Southampton, United Kingdom \\ sw10g13@soton.ac.uk
}

\author{
J. Hemrle, L. Kaufmann \\ ABB Switzerland Ltd., Corporate Research \\ Corporate Research, RD.E4 Segelhofstrasse 1K \\ 5405,Baden- Dättwil, Switzerland
}

\begin{abstract}
This paper investigates the electric potential and field of a two phase (gas-liquid) system. The study uses finite element analysis (FEA) techniques to investigate the impact of a gas bubble on the bulk electric field. The FEA model is expanded to consider the multiple bubble breakdown probability using Monte Carlo techniques. The numerical results demonstrate that compared with bubble quantity, bubble radius is the dominant factor for two phase system electrical breakdown. Furthermore, the effective breakdown strength of a two phase system has been determined as a function of gas phase volume fraction. The predicted two phase effective breakdown strength has then been compared against the mixture rule, where good agreement was achieved.
\end{abstract}

Keywords-thermosiphon, two phase system, thermal bubble, electrical breakdown.

\section{INTRODUCTION}

Nowadays, electrical power devices are following the trend of most other electrical machines by becoming smaller and portable, while at the same time incorporating vast functionality. Generator circuit breakers can be seen as representative of these technological advances. They are widely applied to switch the terminal voltage of the generators and offer better performance than a combined alternator and transformer i.e. a unit connection. However thermal considerations can restrict the rated current of the generator circuit breaker. With the purpose of relieving the thermal limitation, the Thermosiphon is a novel passive cooling concept that can be applied to generator circuit breakers [1]. By using this device the defects of an active cooling system, such as noise and maintenance requirements can be avoided.

The Thermosiphon is a heat pipe, which comprises an evaporator, insulating section and a natural convection condenser. Thermosiphon is hermetically sealed and contains a small amount of working fluid such as hydrofluorether (HFE) and is attached to a heat source. Heat is then extracted from the heat source by the evaporation of the working fluid. Due to the small pressure imbalance, the vapour rises to the condenser which transports latent heat to ambient. The condensed vapour flows back to the evaporator through the insulating section under gravity. This creates a closed loop circulating system to continually cool the target object.
As the insulating section connects the high voltage source and grounded housing it therefore bridges a large electric potential. As generally vapour bubbles have significantly lower dielectric strength than liquid, the formation of bubbles has been recognised as an initial mechanism in the insulation breakdown of dielectric liquids [2].

In this study, the effective breakdown strength is investigated through a series of numerical models. Firstly, this study considers the static electric field around bubble within the working fluid using $2 \mathrm{D}$ and $3 \mathrm{D}$ models. The $2 \mathrm{D}$ model has then been developed further to estimate possible breakdown paths and their associated probability. Finally, an initial approach to predict the effective breakdown strength of the two phase dielectric system is proposed as a function of gas phase volume fraction.

\section{BREAKDOWN PROCESS IN TWO PHASE SYSTEM AND PREVIOUS EXPERIMENTS RESULT}

\section{A. Dielectric fluid boiling behaviour}

The boiling behaviour of the dielectric fluid under electric field can influence the insulation factor significantly. Within the Thermosiphon, the working fluid is not only used as an evaporation medium but also acts as the dielectric liquid to isolate the high voltage. Previous studies have demonstrated boiling behaviour of the dielectric fluid depends on ambient temperature [1], pressure [3] and electrical field [4]. Due to these strongly coupled factors, this initial study does not consider bubble formation or movement and instead considers a "snap shot" at a reasonable distance from the heater electrodes.

\section{B. Two phase system breakdown process}

The two phase system can be viewed as the mixture of gas phase and liquid phase. Due to the different relative permittivity of the liquid $\left(\varepsilon_{1}\right)$ and gas $\left(\varepsilon_{\mathrm{g}}\right)$ the electrical field distributes non-uniformly in the system. This leads to a field enhancement around the bubbles, which increases the possibility of gaseous ionization through the movement of the ions and electrons which may result in a gas discharge. This gaseous discharge phenomenon may cause the dielectric liquid to decompose and increase the probability of entire system breakdown. 


\section{The previous experiments result}

Previous experimental studies using Helium and Nitrogen as the dielectric fluid have shown that the heater power and gas particle radius have a significant influence on the two phase system breakdown phenomenon [2]. Starting with insufficient heat to produce bubbles, the electrical breakdown strength is a product of the dielectric liquid phase only, as expected. As the heater power increases, bubbles are produced, and due to their lower breakdown strength than the liquid phase the breakdown strength between the electrodes decreases. Furthermore, it was also demonstrated that whilst an increased heater power increases the bubble production rate, the application of a potential difference across the electrodes reduces this production rate and causes bubbles which are elongated in the direction of electrical field to be produced. At certain applied voltages, the fixed bubble abruptly grows and bridges the whole space between two electrodes. This is called lock vapour phenomenon. When lock vapour is formed the breakdown voltage of entire block drops rapidly from the liquid value to the gaseous value and leads the whole system breakdown phenomenon occur [5].

\section{ELECTRIC FIELD DISTRIBUTION AROUND BUBBLE}

In order to obtain an appreciation for the electric field around a bubble, the electric potential distribution around a spherical bubble has been analysed using a FEA approach.

The electric potential is predicted through $2 \mathrm{D}$ and $3 \mathrm{D}$ steady state model in between two parallel plate square electrodes, which are separated by $10 \mathrm{~cm}$ and are $2.5 \mathrm{~cm}$ wide. Under the static condition the relationship between electric field $(\boldsymbol{E})$ and electric potential $(\phi)$ within the modelling domains are predicted through the following relationships:

$$
\begin{aligned}
& E=-\nabla \varphi \\
& \varepsilon_{0} \nabla \mathrm{V}=0
\end{aligned}
$$

Where $\varepsilon_{0}$ is the permittivity of vacuum. Inherent within these equations are the assumptions that for this model the space charge density and the electric polarization are 0 . Equation 2 represents Gauss' law.

For this study the bulk material is assumed to be HFE and the dielectric constant of the liquid phase is 7.40 [6]. At present the authors are unable to obtain a relative permittivity value for HFE gas, and so instead the relativity permittivity of helium (1.057) was used [7]. The choice of Helium will not significantly affect the numerical predictions, since relativity permittivity's gasses are generally around 1.0.

Fig. 1 shows the electric potential contours and electric field strength around a single spherical bubble in the middle of two electrode plates when 1MV is applied. The potential contours near to the bubble surface are distorted due to the different dielectric constants. Conversely, the potential contours are distributed uniformly either inside the bubble or away from the bubble, as expected. From the electric field aspect, the maximum electric field is distributed uniformly inside the bubble. However, the electric field is distorted significantly at the bubble surface. The bubble's equatorial plane region achieves peak electric field. Conversely, the minimum electric field is obtained at the two polar area of the bubble. As the result, the maximum electric field gradient appears at the upper side of the bubble.

Finally, by comparing the 2D and 3D simulation results in Fig. 1, it can be seen that the maximum electric field value variation is approximately $15 \%$. Thus, the distribution and numerical values of electric potential and field in $2 \mathrm{D}$ and $3 \mathrm{D}$ model are reasonable comparable. As the 3D model need complex calculation and time consuming further numerical models used in this study were limited to 2D model, which allows simpler analysis and provides a basis for further study as required.
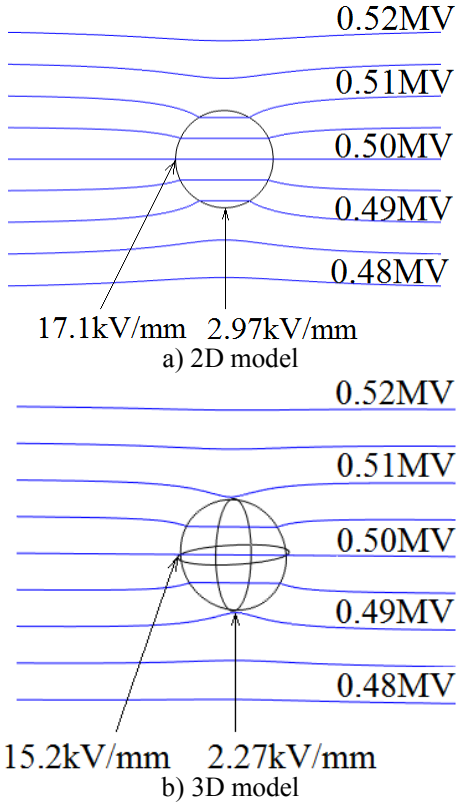

Fig. 1. Electric potential and field around a bubble in the middle of electrode plates (bubble radius $\mathrm{R}=0.75 \mathrm{~mm}$, applied voltage $\mathrm{V}=1 \mathrm{MV}$ ).

\section{MODELLING TWO PHASE SYSTEM BREAKDOWN}

After analysing the electric field around a single bubble, multiple bubble breakdown phenomenon has been analysed by varying bubble radius and bubble quantity.

\section{A. Model assumptions}

For the model there are four assumptions. Firstly, the scale of each bubble (around $1 \mathrm{~mm}$ ) and insulating section $(10 \mathrm{~cm})$ is so significantly different, so to achieve a sufficient gas phase volume fraction for a breakdown to occur, a very large number of bubbles need to be modelled. Such a large number of bubbles makes the model very computational intensive to solve. By assuming that the bubble distribution is uniform within the insulating section, the geometry considered by the model can be a small section of the total insulation section. Secondly, as the relaxation time of HFE is several orders of magnitude higher than the time period of the dynamic process, 
no obvious free charges occur in the HFE and therefore, the Coulomb force can be ignored [4]. Thirdly, as bubble coalescence and collapse occurs over a very short period, these phenomena are neglected in the simulation. Finally, the bubble deformation is not considered and the breakdown path inside the bubble is estimated to be equal to the bubble diameter.

\section{B. Model geometry}

The entire insulating section is divided into ten blocks, $1 \mathrm{~cm}$ high and $2.5 \mathrm{~cm}$ wide. The middle block is the modelled with a $0.1 \mathrm{MV}$ potential applied to its electrodes. Since bubble formation and movement has not been considered within this model, to consider the most realistic bubble distribution, multiple bubbles are positioned randomly within the geometry.

\section{Multiple bubble breakdown prediction method.}

The electric breakdown of the entire two phase system is determined by considering the FEA calculated potential difference profile. Using the potential difference, the results are first interrogated to determine if the electric strength across a bubble exceeds the gaseous dielectric strength that $3.0 \mathrm{kV} / \mathrm{mm}$ and if so a breakdown is assumed to occur. If a breakdown occurs, a perfect conducting channel is assumed to form through the bubble, whereby the electric potential on both sides of the bubble are assumed to be the same. Secondly, the breakdown path between bubbles has been examined. This process determines the electric strength between neighbouring bubbles and if it exceeds the liquid phase dielectric strength which is $15.7 \mathrm{kV} / \mathrm{mm}$ [6] a breakdown path assumed to occur between these bubbles. If the paths emerge, the high voltage transfers continually in the liquid so that breakdown paths may form like a tree from the HV source side to grounded side. Finally, by using a breadth first search to see if the breakdown paths calculated connect the top and bottom. Due to the random bubble distribution a Monte Carlo simulation is used to predict breakdown probability. In this study the breakdown probability is equal to the number of times an entire breakdown is predicted divided by the total number of simulation times. To obtain an accurate breakdown probability, initial studies have shown that the numerical simulations must be repeated 200 at least times.

\section{EXPERIMENTAL RESULTS AND DISCUSSION}

From the introduction in section II, both bubble radius and bubble quantity could change the breakdown probability. To investigate these results numerically, these parameters are varied within the developed model.

\section{A. Varying volume fraction by changing bubble radius.}

To investigate what impact the radius has on breakdown probability, the bubble quantity is fixed and bubble radius is varied from $0.6 \mathrm{~mm}$ to $1.25 \mathrm{~mm}$. A selection of numerical results are presented in Fig. 2.

There are 10 bubbles emerge in the simulation section with $0.06 \mathrm{~mm}$ and $1.25 \mathrm{~mm}$ radii. It indicates that larger bubble radius has higher ability to distort electric potential.

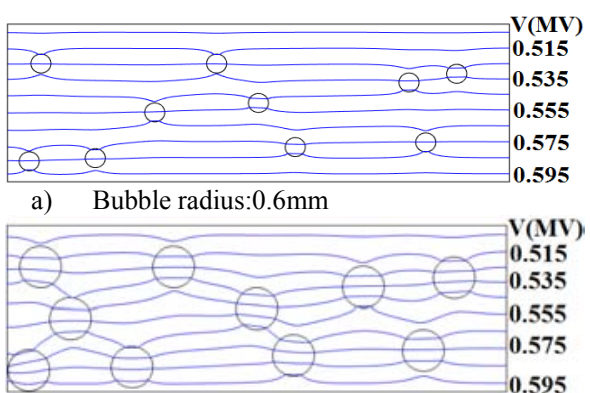

b) Bubble radius: $1.25 \mathrm{~mm}$

Fig. 2. Electric field counters around bubbles.

To investigate the impact of bubble radii further, the breakdown probability for different total number of bubbles is presented in Fig. 3. This figure demonstrates that the system breakdown probability is positively impacted by increasing bubble radius.

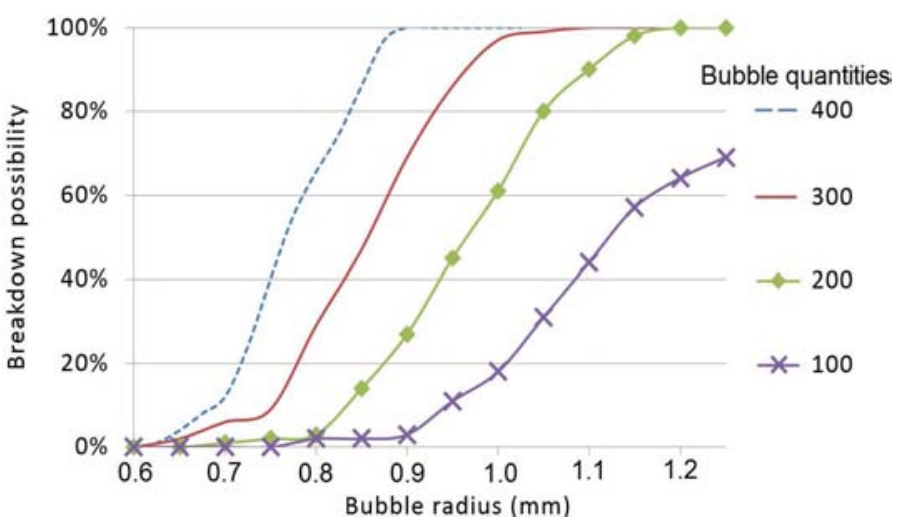

Fig. 3. Bubble radius effect on system breakdown probability.

B. Varying volume fraction by changing bubble quantity.

To investigate the impact of bubble number on the breakdown probability, the bubble radius is fixed and bubble quantity is varied from 0 to 400 .

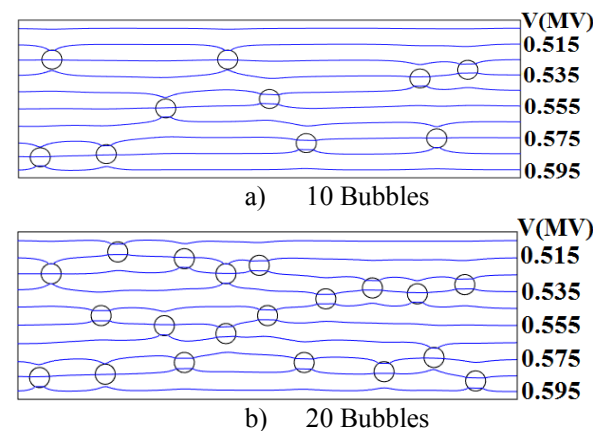

Fig. 4. Electric potential contour around different number bubbles.

From Fig.4 it can be seen that by increasing bubble quantity, the electric potential is non-uniform and distorted more acutely.

The simulation results presented in Fig.5 shows that by fixing bubble radius, the system breakdown probability is also positively impacted by the amount of bubble. Decreasing the 
bubble radius reduces the rate at which breakdown probability increases with bubble number.

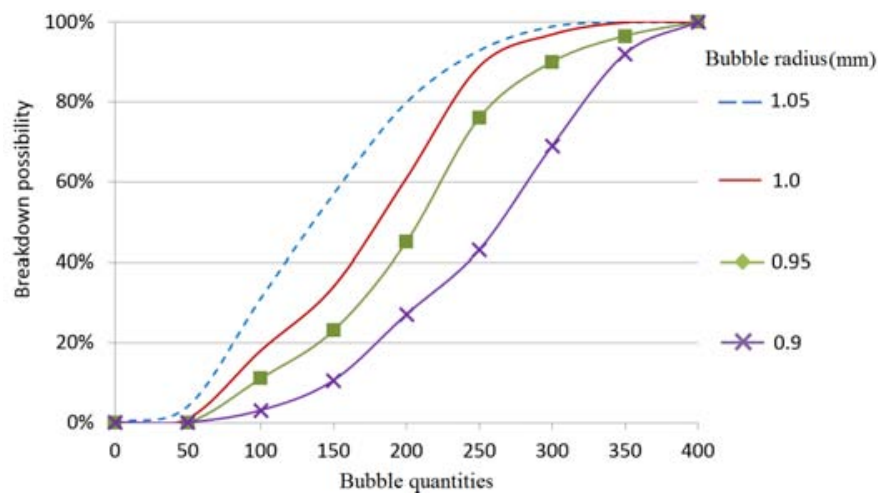

Fig. 5. Bubble quantity effect on system breakdown probability.

\section{The dominant factor}

The previous sections have shown that both bubble radius and bubble quantity have an impact on the breakdown probability and so it is important to investigate, which of these factors dominates. Fig. 6 indicates the relationship between bubble volume fractions with breakdown probability numerically. In the $2 \mathrm{D}$ model the bubble volume fraction is represented by the total gas phase area divided by total area of the model. Furthermore the gas phase area is equal to the bubble area times bubble quantity. Hence, simulation results demonstrate that with same bubble volume fraction, few larger bubbles have a higher breakdown probability. As a consequence, bubble radius is the dominant factor in two phase system electrical breakdown phenomenon.

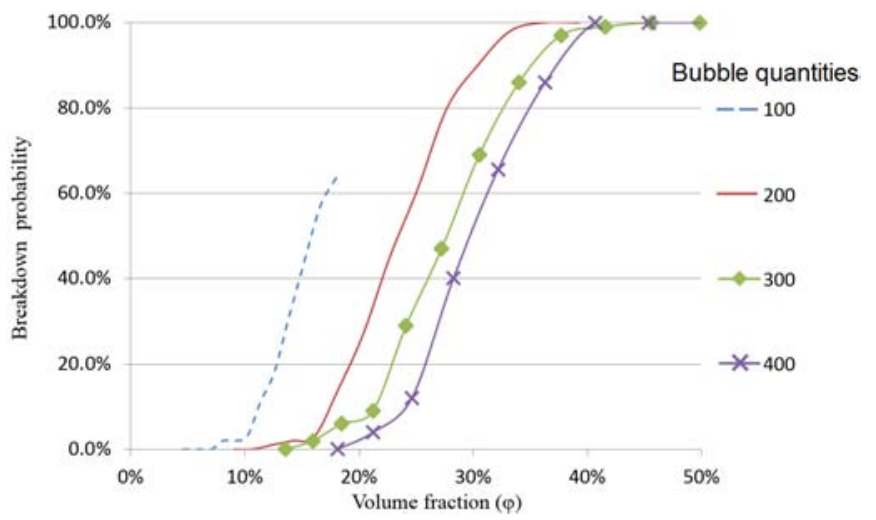

Fig. 6. Gas volume fraction effect on breakdown probability.

\section{Comparison between FEA result and mixture rule}

Previous published studies have used the Mixture rule, which is a weighted volume fraction mean to predict the bulk material properties [8]. In this method the equivalent dielectric strength is represent as the function with the bubble volume fraction. By applying different voltage and corresponding breakdown bubble volume fraction the FEA dielectric strength can be plotted as shown in Fig.7. It can be seen that the FEA breakdown strength has agreement with values calculated using the Mixture rule. Where the FEA model predicts a lower breakdown strength for mixtures of liquid/gas.

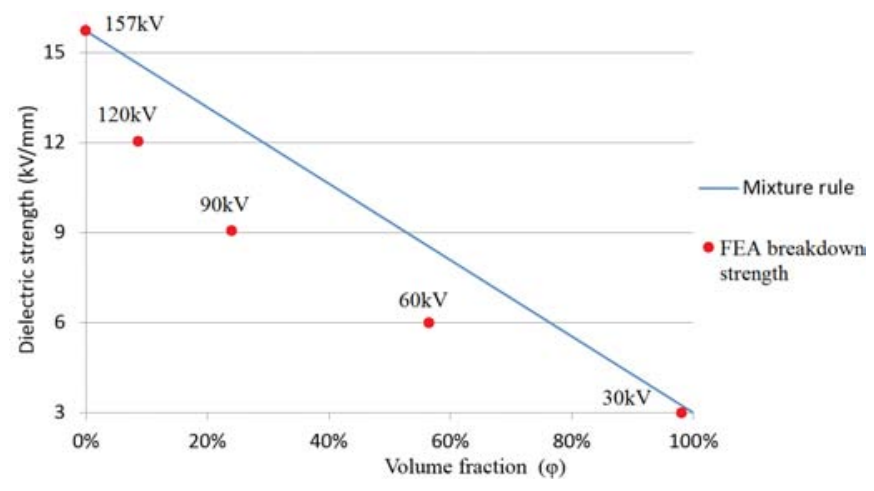

Fig. 7. Comparison FEA breakdown strength with Mixture rule.

\section{CONCLUSION}

A numerical study has found that the electric field is distorted greatly due to the presence of the thermal bubbles. The 2D and 3D FEA results are shown to be comparable. Therefore, using a 2D Monte Carlo method, the breakdown probability for the two phase system can be investigated. The numerical results give good agreement with previous experiments where bubble radius and quantity are shown to reduce the two phase system breakdown voltage. Furthermore, it has been demonstrated that the bubble radius is the dominant factor, which indicates that few larger bubbles are more likely cause two phase system breakdown.

\section{REFERENCE}

[1] D. Braun, G. Cavaliere, K. Dahinden, and M. Lakner, "Heat Pipes - A Novel Cooling Principle for Generator Circuit-breakers," in POWER PLANTS 2009, 2009, pp. 69-72.

[2] M. Hara, H. Nakagawa, J. Suehiro, and T. Shinohara, "Electrical breakdown triggered by a free conducting spherical particle in saturated liquid He I and He II under uniform DC field," IEEE Trans. Dielectr. Electr. Insul., vol. 10, no. 6, pp. 1022-1031, Dec. 2003.

[3] H. Frid and M. Becerra, "Simulation of microbubbles during the initial stages of breakdown in cyclohexane," 2013 Annu. Rep. Conf. Electr. Insul. Dielectr. Phenom., pp. 901-904, Oct. 2013.

[4] P. Wang, D. J. Swaffield, P. L. Lewin, and G. Chen, "Thermal Bubble Motion in Liquid Nitrogen under Non- uniform Electric Fields," pp. 626-634.

[5] M. Hara, T. Kaneko, and K. Honda, "Thermal-bubble initiated breakdown characteristics of liquid helium and nitrogen at atmospheric pressure," IEEE Trans. Electr. Insul., vol. 23, no. 4, pp. 769-778, 1988.

[6] "3M TM Novec TM Engineered Fluid HFE-7100 for Heat Transfer." [Online]. http://multimedia.3m.com/mws/media/654950/3mtm-thermalmanagement-fluids.pdf?fn=bro_heattrans.pdf. [Accessed: 01-Jan-2002].

[7] William M. Haynes, CRC Handbook of Chemistry and Physics, 95th Edition, 95th ed. CRC Press, 2014, pp. 6-209.

[8] K. Wakino, "A new proposal on mixing rule of the dielectric constant of mixture," Proc. 1994 IEEE Int. Symp. Appl. Ferroelectr., pp. 33-38, 1994. 